\title{
ARTíCULO \\ Biomagnificación de mercurio en la cadena trófica del Delfín Moteado del Atlántico (Stenella frontalis), usando el isótopo estable de nitrógeno como marcador ecológico
}

Mercury biomagnification in the Atlantic spotted dolphin (Stenella frontalis) food chain, using nitrogen stable isotope as an ecological tracer

\author{
Helena A. Kehrig ${ }^{*}$, Gilberto Baptista ${ }^{2}$, Ana Paula M. Di Beneditto ${ }^{1}$, \\ Marcelo G. Almeida ${ }^{1}$, Carlos E. Rezende ${ }^{1}$, Salvatore Siciliano ${ }^{3}$, \\ Jailson F. de Moura ${ }^{4}$ y Isabel Moreira ${ }^{2}$
}

\author{
${ }^{1}$ Laboratório de Ciências Ambientais, Universidade Estadual do Norte Fluminense, Campos dos Goytacazes, RJ 28013-602, Brasil. \\ helena.kehrig@pq.cnpq.br \\ ${ }^{2}$ Departamento de Química, Pontifícia Universidade Católica do Rio de Janeiro, RJ 22453-900, Rio de Janeiro, Brasil \\ ${ }^{3}$ Instituto Oswaldo Cruz (IOC/Fiocruz), Pavilhão Mourisco - sala 122, Manguinhos, Rio de Janeiro, RJ, 21040-360, Brasil \\ ${ }^{4}$ Leibniz Center for Tropical Marine Ecology, ZMT, Bremen 28359, Germany
}

\begin{abstract}
An assessment of mercury $(\mathrm{Hg})$ concentrations and nitrogen stable isotope $\left(\delta^{15} \mathrm{~N}\right)$ was conducted in the food chain of the Atlantic spotted dolphin (Stenella frontalis), including phytoplankton, zooplankton, planktivorous fish and its major prey (predatory fish and a single species of cephalopod), that compose a tropical trophic chain of the Brazilian southeastern coast. Tissue concentrations of $\mathrm{Hg}$ in a voracious predator fish, the largehead hairtail (Trichiurus lepturus), one of the dolphin's prey, were 9.8 times lower than median concentrations found in dolphin tissues. $\delta^{15} \mathrm{~N}$ values in predatory fish were found to be lower to those of its predator the spotted dolphin. Isotopic data suggested significant differences for $\delta^{15} \mathrm{~N}$ along the trophic chain, with the top predator (dolphin) exhibiting heavier value, followed by the voracious predator fish and the benthonic carnivorous fish, the whitemouth croaker (Micropogonias furnieri). Phytoplankton displayed the lightest $\delta^{15} \mathrm{~N}$, followed by zooplankton and the planktophagous fish, the lebranche mullet (Mugil liza). This fish species and the cephalopod showed the lowest median Hg concentration. All links of the entire trophic chain presented trophic transfer of $\mathrm{Hg}$ with a biomagnification factor higher than 1. A significant relationship was found between the log $\mathrm{Hg}$ concentration and trophic level (TL) of all evaluated species, with a positive slope $(\beta=0.87)$. The calculated trophic magnification factor ( $T M F=7.44$ ) indicates that $\mathrm{Hg}$ concentration increased per $\mathrm{TL}$, and also that the entire coastal food chain from the South Atlantic Ocean presented a biomagnification power of $\mathrm{Hg}$ within a range previously reported for tropical coastal ecosystems.
\end{abstract}

Key words: Marine mammal, coastal aquatic biota, toxic element, biotransference, ecological tracer

Resumen.- Se realizó una evaluación de las concentraciones del mercurio $(\mathrm{Hg})$ y del isótopo estable de nitrógeno $\left(\delta^{15} \mathrm{~N}\right)$ en la cadena trófica del Delfín Moteado del Atlántico (Stenella frontalis), incluyendo fitoplancton, zooplancton, peces planctívoros y sus principales presas (peces depredadores y una sola especie de cefalópodo), componentes de una cadena trófica tropical de la costa sureste de Brasil. Una de las presas del delfín, un pez depredador voraz, el pez sable (Trichiurus lepturus), presentó una concentración mediana del $\mathrm{Hg} 9,8$ veces inferior que la del delfín. El valor de $\delta^{15} \mathrm{~N}$ es más bajo que al de su depredador. Los datos del isótopo han sugerido que ha habido diferencias significativas para el $\delta^{15} \mathrm{~N}$ a lo largo de la cadena trófica, en la que en el mayor depredador (delfín) se ha mostrado más pesado, seguido por el pez depredador y el pez bentófago, la corvina (Micropogonias furnieri). Se ha observado que el fitoplancton presentó el $\delta^{15} \mathrm{~N}$ más liviano, seguido por el zooplancton y el pez planctófago, el lebranche (Mugil liza). Este pez y el cefalópodo han mostrado la mediana más baja de Hg. Se han encontrado transferencias mayores que 1 en todos los vínculos de la cadena evaluada. Se ha obtenido una relación significativamente positiva entre la concentración del Hg (en logaritmo) y el nivel trófico ( $T L$ ), incluyendo todas las especies investigadas, presentando una pendiente positiva $(\beta=0,87)$. El factor de magnificación trófica $(F M T=7,44)$ indica que la concentración del Hg ha aumentado por TL, y también que toda la cadena trófica costera presentó un poder de biomagnificación de $\mathrm{Hg}$ dentro de un rango previamente reportado para ecosistemas costeros tropicales.

Palabras clave: Mamífero marino, biota acuática costera, elemento tóxico, trazador ecológico, biotransferencia 


\section{INTRODUCCIÓN}

El mercurio (Hg) es un contaminante global reconocido como un gran problema para el medio ambiente y los océanos, que conlleva preocupación y afecta en distintos grados la fauna marina y la salud de los seres humanos (UNEP 2013).

El Hg en su forma orgánica más tóxica, el metilmercurio (MeHg), se acumula en los tejidos de los organismos a lo largo de su vida (Baeyens et al. 2003), debido a su habilidad en cruzar las membranas celulares, resultando en gran absorción, propensión para el sistema nervioso y mayor retención dentro del cuerpo (Neathery \& Miller 1975). El MeHg se bioacumula hasta un millón de veces a lo largo de la cadena trófica acuática, desde la base (microorganismos) hasta los organismos de la parte superior de la cadena (peces depredadores y mamíferos), por adsorción a la superficie corporal y, principalmente, por ingestión de alimentos (Kehrig et al. 2009a). Así, el MeHg es el principal responsable por la acumulación del $\mathrm{Hg}$ en los organismos acuáticos y también, por su transferencia de un nivel trófico a otro. En vista de su capacidad en acumularse en los organismos marinos a través de los niveles tróficos, se espera que las concentraciones más elevadas sean observadas en los peces predadores y en los mamíferos marinos, o sea, en los niveles tróficos superiores en las cadenas y redes tróficas marinas, a través del proceso de biomagnificación (Kehrig et al. 2009a).

Sin embargo, las concentraciones de $\mathrm{Hg}$ en las especies marinas no dependen solamente de la contaminación del medio ambiente, sino que también, reciben la influencia de diversos parámetros ecológicos y biológicos, como el hábito alimenticio, el nivel trófico, la edad, el sexo, el estado de madurez sexual, la exposición a Hg y especiación química del Hg (Das et al. 2004, Panebianco et al. 2011). La transferencia trófica a lo largo de las cadenas y redes tróficas marinas es una importante herramienta para evaluar el proceso de biomagnificación del $\mathrm{Hg}$ de un nivel trófico a otro, o sea, desde la presa hasta el consumidor (Barwick \& Maher 2003).

En las últimas décadas, los isótopos estables han proporcionado datos complementarios a respecto de la ecología trófica de los organismos. El isótopo estable del nitrógeno $\left(\delta^{15} \mathrm{~N}\right)$ es ampliamente utilizado para estimar el nivel trófico que ocupan los depredadores en las cadenas y redes tróficas marinas, una vez que el $\delta^{15} \mathrm{~N}$ de un consumidor refleja los contenidos de nitrógeno de sus presas (De Niro \& Epstein 1981, Peterson \& Fry 1987). La incorporación del $\delta^{15} \mathrm{~N}$ en el depredador es función de la concentración de la presa consumida, resultando en un enriquecimiento de este isotopo a través de las cadenas y redes tróficas (De Niro \& Epstein 1981, Cabana \& Rasmussen 1994) que puede variar dependiendo del depredador o consumidor. $\mathrm{El} \delta^{15} \mathrm{~N}$ es un importante indicador en la ecología alimentaria y puede relacionarse a la exposición de los organismos ante los contaminantes (Jardine et al. 2006, Aubail et al. 2011, Di Beneditto et al. 2011, Kehrig et al. 2013).

En este estudio se evaluaron las concentraciones de Hg en el tejido muscular del Delfín Moteado del Atlántico Stenella frontalis (Cuvier, 1829) y también, en algunas de sus presas, cefalópodo (Doryteuthis plei) y peces (Trichiurus lepturus, Micropogonias furnieri, Sardinella brasiliensis, Mugil liza), que componen una cadena trófica costera tropical. Los principales objetivos fueron relacionar las concentraciones de $\mathrm{Hg}$ con la estructura trófica de la cadena trófica usando el isótopo estable $\left(\delta^{15} \mathrm{~N}\right)$ como biomarcador y también, comparar las posibles transferencias tróficas y la magnificación trófica del Hg en la cadena, desde las presas hasta el delfín. Se utilizó el factor de magnificación trófica (FMT) como una herramienta para evaluar y cuantificar la biomagnificación del Hg mediante su transferencia, a través de la dieta, para los consumidores a lo largo de la cadena trófica.

\section{MATERIALES Y MÉTODOS}

\section{Área de ESTUdio}

Entre el 2003 y 2012, se tomaron muestras de ejemplares de delfín, Stenella frontalis, provenientes de la captura accidental en redes de pesca y también, de algunas de sus presas, en la

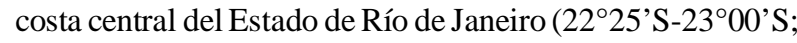
Fig. 1). Esta región, en el sureste de Brasil, se caracteriza por eventos de surgencia (upwelling) que proveen una fuente de alimento para las especies superiores en la red trófica costera, tales como los delfines (Coelho-Souza et al. 2012).

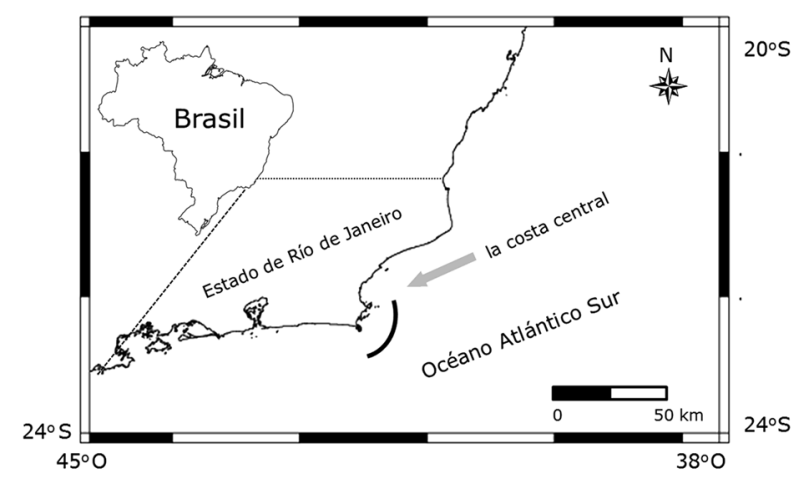

Figura 1. Localización de la región de muestra a lo largo de la costa central del Estado de Río de Janeiro / Localization of the sampling region at Rio de Janeiro's central coast 


\section{ESPECIES INVESTIGADAS Y PREPARACIÓN DE LAS MUESTRAS}

El Delfín Moteado del Atlántico es un pequeño cetáceo dentado que se distribuye en aguas tropicales del océano Atlántico y, en menor grado, en aguas templadas del hemisferio sur (Amaral et al. 2015). Como la mayoría de las especies de delfines costeros, $S$. frontalis vive en aguas poco profundas cerca de la costa $(<200 \mathrm{~m})$, aunque este delfín también puede ser visto en aguas más profundas (200-2000 m) (Mullin \& Fulling 1998). En el área de estudio, este cetáceo se alimenta de cefalópodos y peces que viven en hábitats costeros y algunos de ellos están asociados con las zonas de estuarios. Las presas, el cefalópodo (D. plei) y los peces (T. lepturus, M. furnieri, S. brasiliensis, M. liza), son especies abundantes a lo largo del año en la región del presente estudio (Di Beneditto et al. 2001, Melo et al. 2010, Lopes et al. 2012). Después de la identificación de las especies ya citadas, se les tomó el peso y la longitud corporal (Tabla 1) y se extrajo una muestra sin piel de los tejidos musculares, según los procedimientos de la FAO/ SIDA (1983).

Previo a la disección de muestras de tejido muscular (la porción dorsal posterior) de ejemplares de delfín, se determinó el sexo y se tomaron medidas de longitud. Todos los ejemplares de delfín examinados eran de individuos inmaduros de ambos sexos. El estado de madurez sexual de cada individuo se estimó a partir de su medida de longitud, de acuerdo con el estudio reportado por Siciliano et al. (2007), según el cual los individuos adultos, o sea, maduros, poseen más de $223 \mathrm{~cm}$ de longitud.

Para componer la cadena trófica del Delfín Moteado del Atlántico, desde su nivel más alto (delfín) hasta su nivel más bajo (i.e., base de la cadena trófica), se utilizaron los datos de las muestras de fitoplancton (Chlorococcales spp. y diatomeas), zooplancton (copépodos y cladóceros) y también, los de los ejemplares de camarón (Xiphopenaeus kroyeri), que fueron recolectados para un estudio previo desarrollado en una región costera cercana (Di Beneditto et al. 2012).

Tabla 1. Nombres científicos y comunes, hábito alimentario, longitud y número de ejemplares ( $\mathrm{N}$ ), la mediana y el rango (mín-máx) de las concentraciones, en peso seco, de Hgy el rango (mín-máx) de la composición del isótopo estable $\delta^{15} \mathrm{~N}$ (\%o) en el tejido muscular y el nivel trófico (TL) de todas las especies / Scientific and common names, feeding habits, length and number of specimens, and the median and range of $\mathrm{Hg}$ concentrations (on dry weight basis) and $\delta^{15} \mathrm{~N}$ stable isotope composition (\%o) in the muscle tissue and trophic level (TL) of all species

\begin{tabular}{|c|c|c|c|c|c|c|}
\hline Especie & \multicolumn{4}{|c|}{ Nombre común } & \multicolumn{2}{|c|}{ Hábito alimentario } \\
\hline $\begin{array}{l}\text { Stenella frontalis } \\
\text { Trichiurus lepturus } \\
\text { Micropogonias furnieri } \\
\text { Sardinella brasiliensis } \\
\text { Mugil liza } \\
\text { Doryteuthis plei } \\
\text { Xiphopenaeus kroyeri } \\
\text { Especies agrupadas } \\
\text { Especies agrupadas }\end{array}$ & \multicolumn{3}{|c|}{$\begin{array}{l}\text { Delfín Moteado del Atlántico } \\
\text { pez sable } \\
\text { corvina } \\
\text { sardinella brasileña } \\
\text { lebranche } \\
\text { calamar costero púrpura } \\
\text { camarón }\end{array}$} & $\begin{array}{l}\text { cetáceo } \\
\text { pez } \\
\text { pez } \\
\text { pez } \\
\text { pez } \\
\text { cefalópodo } \\
\text { crustáceo } \\
\text { zooplancton } \\
\text { fitoplancton }\end{array}$ & \multicolumn{2}{|c|}{$\begin{array}{l}\text { depredador voraz } \\
\text { depredador voraz } \\
\text { bentófago } \\
\text { planctófago } \\
\text { planctófago } \\
\text { carnívoro } \\
\text { omnívoro } \\
\text { consumidor primario } \\
\text { productor primario }\end{array}$} \\
\hline Especie & $\begin{array}{c}\text { Longitud } \\
(\text { rango en } \mathrm{cm} \text { ) }\end{array}$ & $\mathrm{N}$ & \multicolumn{2}{|c|}{$\begin{array}{l}{[\mathrm{Hg}] \mu \mathrm{g} \mathrm{g}^{-1} \text { p.s. }} \\
\text { mediana (rango) }\end{array}$} & $\begin{array}{c}\delta^{15} \mathrm{~N}(\% o) \\
\text { rango }\end{array}$ & $\mathrm{TL}$ \\
\hline Stenella frontalis & $166-200$ & 7 & \multicolumn{2}{|c|}{$3,32(1,02-4,23)$} & $13,2-16,4$ & 4,0 \\
\hline Trichiurus lepturus & $105-148$ & 12 & \multicolumn{2}{|c|}{$0,34(0,22-0,81)$} & $12.7-14,3$ & 3,6 \\
\hline Micropogonias furnieri & $47-57$ & 10 & \multicolumn{2}{|c|}{$0,35(0,23-1,25)$} & $13,2-14,1$ & 3,7 \\
\hline Sardinella brasiliensis & $24-26$ & 10 & \multicolumn{2}{|c|}{$0,15(0,10-0,20)$} & $10,6-11,5$ & 2,9 \\
\hline Mugil liza & $53-58$ & 10 & \multicolumn{2}{|c|}{$0,057(0,017-0,11)$} & $7,7-9,0$ & 2,2 \\
\hline Doryteuthis plei & $15-19$ & 10 & \multicolumn{2}{|c|}{$0,047(0,034-0,060)$} & $10.8-11,6$ & 3,0 \\
\hline Xiphopenaeus kroyeri & $8-13$ & 1 & \multicolumn{2}{|r|}{$0,079^{\mathrm{a}}$} & $10,3^{\mathrm{a}}$ & 2,7 \\
\hline Especies agrupadas & $160 \mu \mathrm{m}$ & 1 & \multicolumn{2}{|r|}{$0,0063^{\mathrm{a}}$} & $7,9^{\mathrm{a}}$ & 2,0 \\
\hline Especies agrupadas & $25 \mu \mathrm{m}$ & 1 & \multicolumn{2}{|r|}{$0,0042^{\mathrm{a}}$} & $5,2^{\mathrm{a}}$ & 1,2 \\
\hline
\end{tabular}

${ }^{\mathrm{a}}$ : valores de la concentración de $\mathrm{Hg}$ y el $\delta^{15} \mathrm{~N}$ (Di Beneditto et al. 2012) 
Las muestras de músculo de todas las especies (perdieron cerca de $70 \%$ del contenido de agua) fueron liofilizadas y almacenadas hasta que la determinación analítica del $\mathrm{Hg}$ e isótopo estable $\left(\delta^{15} \mathrm{~N}\right)$ fuese hecha. Los nombres científicos, el número de ejemplares, el hábito alimenticio y el rango de longitud de las especies se encuentran en la Tabla 1.

\section{Análisis del mercurio (HG)}

Para la determinación analítica de $\mathrm{Hg}$, se realizó una mineralización del tejido seco (100 mg) en un medio con una mezcla de ácidos fuertes (nítrico-sulfúrico). El método analítico fue la espectroscopia de absorción atómica con vapor frío, sistema FIMS, utilizando $\mathrm{NaBH}_{4}$ como agente reductor. Una descripción más detallada del método utilizado se encuentra en Kehrig et al. (2009b). A fin de cumplir con los estándares de calidad analítica, se utilizaron un control en blanco, dos réplicas y material de referencia certificado DORM-2 (Hg: 4,64 $\pm 0,26$ $\left.\mu \mathrm{g} \mathrm{g}^{-1}\right)$ del Consejo Superior de Investigaciones CientíficasCanadá. Los valores de recuperación fueron siempre $\geq 90 \%$ de los valores certificados. La reproductibilidad se evaluó usando el coeficiente de variación de las réplicas, que fueron menores que $10 \%$. Los valores encontrados para el $\mathrm{Hg}$ fueron $4,59 \pm 0,12 \mu \mathrm{g} \mathrm{g}^{-1}$.

El límite de detección (LOD) fue calculado de acuerdo con la IUPAC, basándose en la desviación estándar (s) de las lecturas de 10 de los blancos del método y la pendiente de la curva de calibración ( $\mathrm{LD}=3 \mathrm{~s} /$ pendiente). Con base en los resultados obtenidos para $\mathrm{LD}\left(0,02 \mu \mathrm{g} \mathrm{L}^{-1}\right)$, se calculó la cantidad mínima posible de ser determinada del $\mathrm{Hg}$ en $100 \mathrm{mg}$ de tejido analizado, mediante el uso del método en cuestión, obteniéndose el valor de $0,0028 \mu \mathrm{gHg} \mathrm{g}^{-1}$.

Los datos de las concentraciones de $\mathrm{Hg}$, en las muestras de fitoplancton, zooplancton y también en los de los ejemplares de camarón, se hallan en Di Beneditto et al. (2012).

\section{ANÁlisis del isótOPo ESTABLE de NITRÓGENO $\left(\delta^{15} \mathrm{~N}\right)$}

Para la determinación del isótopo estable de nitrógeno en las muestras de músculo liofilizadas en polvo fino, de todas las especies (delfín, cefalópodo y peces), se utilizó un espectrómetro de masas de razón isotópica Advantage Delta V (Thermo Scientific) en interface con Conflo IV, conectado a un analizador elemental flash 2000 (Thermo Scientific). Las proporciones de isótopos estables se expresaron en notación $\delta$ como partes por mil (\%o) de las desviaciones de las normas internacionales de calibración. La razón de nitrógeno se expresa en relación al nitrógeno atmosférico. Se analizaron las muestras con pequeños recipientes vacíos y material de referencia certificada de urea del IVA Analysentechnik $330802174\left(\mathrm{CH}_{4} \mathrm{~N}_{2} \mathrm{O} \mathrm{M}_{\mathrm{w}}=60, \mathrm{~N}=\right.$
$46 \%)$ con la composición isotópica certificada $\left(\delta^{15} \mathrm{~N}=\right.$ $-0,73 \%$ ). A fin de cumplir con los estándares de calidad analítica, se utilizó el material de referencia certificado de isótopos (proteína estándar OEA con composición isotópica certificada, $\delta^{15} \mathrm{~N}=5,9 \%$ ) a cada 10 análisis. La reproductibilidad basada en el análisis triple de $10 \%$ de las muestras fue $\pm 0,2 \%$ o para $\delta^{15} \mathrm{~N}$.

Los datos de $\delta^{15} \mathrm{~N}$ en las muestras de fitoplancton, zooplancton y también en los de los ejemplares de camarón se hallan en Di Beneditto et al. (2012).

El nivel trófico (TL) del Delfín Moteado del Atlántico, de algunas de sus presas, cefalópodo y peces, y también de los ejemplares de camarón, zooplancton y fitoplancton fue estimado en relación al consumidor primario, zooplancton, de la base de la cadena trófica de la costa central del Estado de Río de Janeiro. Se utilizó la ecuación modificada $\left(\mathrm{TL}_{\text {consumidor }}=[2+\right.$ $\left.\left(\delta^{15} \mathrm{~N}_{\text {consumidor }}-\delta^{15} \mathrm{~N}_{\text {consumidor primario }}\right)\right] / 3,4 \%$ ) de Vander Zanden et al. (1997) para estimarse la TL de cada una de las especies. En la ecuación, el consumidor primario ocupa la posición 2 $\left(\mathrm{TL}_{\text {consumidor primario }}=2\right.$ ), en cuanto $\mathrm{TL}_{\text {consumidor }}$ y $\delta^{15} \mathrm{~N}_{\text {consumidor }}$ son la TL y el $\delta^{15} \mathrm{~N}$ de cada especie estudiada, respectivamente, y $3,4 \%$ o representa el factor de discriminación trófica más frecuente para el $\delta^{15} \mathrm{~N}$ utilizado por los organismos acuáticos (Lavoie $e t$ al. 2013).

La regresión lineal simple entre los valores de $\mathrm{Hg}$ y $\delta^{15} \mathrm{~N}$ $\left(\log [\mathrm{Hg}]=\beta^{*}\left(\delta^{15} \mathrm{~N}\right)+\alpha\right)$ para todas las especies (fitoplancton, zooplancton, camarones, cefalópodo, peces y el delfín), a lo largo de la cadena trófica, fue usada para cuantificar la magnificación trófica del $\mathrm{Hg}$, o sea, la variación media de la concentración del Hg con el nivel trófico (Lavoie et al. 2013). La pendiente $\beta$ de la regresión lineal simple es la pendiente de la magnificación trófica y $\alpha$ es la intersección con el eje vertical.

Sin embargo, según Lavoie et al. (2013), el proceso de biomagnificación no se ve solamente afectado por el proceso de la cadena trófica (por ejemplo, la tasa de crecimiento, la diversidad de especies y la longitud de la cadena trófica), sino también, por variables fisicoquímicas ambientales. Por lo tanto, la biomagnificación del $\mathrm{Hg}$ también se puede evaluar usando el nivel trófico (TL) en lugar de los datos de $\delta^{15} \mathrm{~N}$ (Lavoie et al. 2013).

El factor de magnificación trófica (FMT), calculado como el antilog de la pendiente $(\beta)$ de la regresión lineal simple entre el logaritmo de la concentración del $\mathrm{Hg}$ y el TL $\left(\mathrm{FMT}=10^{\beta}\right)$, representa la biomagnificación media por TL a lo largo de la cadena trófica (Borgå et al. 2012). Cuando el FMT es mayor que uno (i.e., FMT > 1 y $\beta>0$ ) indica que el $\mathrm{Hg}$ biomagnifica en la cadena trófica. Si el FMT resulta menor que uno (i.e., 
FMT $<1$ y $\beta<0)$ indica que ocurre una dilución trófica del $\mathrm{Hg}$, aunque el FMT $=1(\beta=0)$ indica que no hay cambios en las concentraciones del $\mathrm{Hg}$ entre los organismos de la cadena trófica (Borgå et al. 2012).

También, se ha usado la razón de las concentraciones de $\mathrm{Hg}$ entre el consumidor y la presa $\left([\mathrm{Hg}]_{\text {consumidor }} /[\mathrm{Hg}]_{\text {presa }}=\right.$ FBM) para evaluar el factor de biomagnificación (FBM o BMF) (Alava \& Gobas 2012, Kehrig et al. 2013), o sea, la transferencia del $\mathrm{Hg}$ desde la presa hasta el consumidor asumiendo el estado estacionario. Cuando el FBM es mayor que uno (i.e., FBM > 1), expresa que hay una transferencia positiva o biomagnificación; pero si el FBM es menor que uno (i.e., FBM < 1), indica que no hay biomagnificación. Sin embargo, el proceso de biomagnificación solamente ocurre cuando hay dos transferencias positivas seguidas a través de por lo menos dos niveles tróficos en una cadena o red trófica (Barwick \& Maher 2003).

\section{Análisis estadísticos}

Todos los análisis estadísticos se realizaron utilizando el programa Statistica ${ }^{\circledR} 7.0$ para Windows (StatSoft, Inc. 19842004, EE.UU.). Se utilizó la estadística descriptiva para determinar los valores medios de triplicados de cada muestra (blancos analíticos, CRM) y los de las medianas de las concentraciones de $\mathrm{Hg}$, así como para la determinación de la desviación estándar y el coeficiente de variación para cada análisis triple de muestras. Se evaluó la normalidad de la distribución de los datos y la homogeneidad de la varianza a través de las pruebas de Shapiro-Wilk. Debido a que los datos no reunieron los criterios de distribución normal, se realizaron pruebas estadísticas no paramétricas. El análisis de varianza se aplicó mediante la prueba de Kruskal-Wallis ANOVA seguido de una prueba post-hoc (Mann-Whitney U-test) para evaluar la existencia de diferencias significativas en las concentraciones de $\mathrm{Hg}, \delta^{15} \mathrm{~N}$ y TL a lo largo de la cadena trófica costera. Se aplicó el coeficiente de correlación por rangos de Spearman $\left(r_{s}\right)$ para determinar la relación entre el logaritmo de las concentraciones de $\mathrm{Hg}$ con el isótopo estable de $\delta^{15} \mathrm{~N}$ y también, con el nivel trófico (TL). Se consideró como nivel de significancia estadística $P \leq 0,05$ para todas las pruebas realizadas.

\section{Resultados}

No se encontró diferencia significativa (prueba $\mathrm{U}, P>0,05$ ) según el sexo en las concentraciones de $\mathrm{Hg}$ y de $\delta^{15} \mathrm{~N}$ en el tejido muscular del Delfín Moteado del Atlántico. Las concentraciones de $\mathrm{Hg}$ aumentaron de manera lineal con la longitud $(\mathrm{L})$ del delfín $\left([\mathrm{Hg}]=0,094 * \mathrm{~L}-14,52, \mathrm{r}_{\mathrm{s}}=0,91, P<\right.$ 0,001).
Se encontraron diferencias significativas $(\mathrm{H}=29,5 ; P<$ 0,0001 ) en las concentraciones de $\mathrm{Hg}$ entre todas las especies, desde el fitoplancton, el zooplancton, los camarones, el cefalópodo y los peces hasta el delfín. Los niveles de $\mathrm{Hg}$ (Tabla 1) aumentaron a medida que se asciende en la cadena trófica; siendo mayor en los ejemplares del delfín, y en contraposición, las más bajas concentraciones se observaron en el fitoplancton (Tabla 1). Sin embargo, las 2 especies de pez, M. furnieri (bentófago) y T. lepturus (depredador voraz) presentaron concentraciones semejantes (prueba $\mathrm{U}, P>0,05$ ) en el tejido muscular, así como las concentraciones observadas en el cefalópodo y pez planctófago (M. liza) (prueba $\mathrm{U}, P>0,05)$.

Se encontraron transferencias positivas en todos los vínculos (9) de la cadena evaluada (Fig. 2). Además, en las 2 cadenas tróficas investigadas, 2 de los múltiples niveles tróficos presentaron transferencias positivas, como por ejemplo: la del cefalópodo (D. plei) para el pez (T. lepturus) y la de este para el delfín, y también, la del zooplancton para el pez ( $S$. brasiliensis), la de este para el pez (T. lepturus) y así como, la de este último para el delfín.

Se encontraron diferencias significativas (prueba $\mathrm{U}, P<$ 0,05), según el nivel trófico (TL) y la composición del isótopo estable de $\delta^{15} \mathrm{~N}$, en el tejido muscular del Delfín Moteado del Atlántico y en su mayor presa, el del pez depredador voraz, $T$. lepturus.

Los valores del $\delta^{15} \mathrm{~N}$ en las muestras de músculo del delfín no se relacionaron significativamente con su longitud $(P>0,05)$, ni con las concentraciones del $\mathrm{Hg}(P>0,10)$. No obstante, los valores del isótopo presentaron una tendencia al aumento con la L y también con el $\mathrm{Hg}$.

Asimismo, se verificaron diferencias significativas $(\mathrm{H}=27,16$, $P<0,001)$ en los valores del $\delta^{15} \mathrm{~N}$ y del TL en las especies, a lo largo de la cadena trófica de la costa central del Estado de Río de Janeiro (Tabla 1). Se encontraron los valores mayores del $\delta^{15} \mathrm{~N}$ y TL en el delfín, seguido por el pez depredador voraz y el pez bentófago (M. furnieri), que incluso se revelaron significativamente semejantes (prueba $\mathrm{U}, P>0,05$ ). El fitoplancton ha mostrado el $\delta^{15} \mathrm{~N}$ más liviano entre todos los componentes de la cadena trófica, ocupando el nivel trófico más bajo (Tabla 1). A este le sigue el zooplancton y la especie de pez planctófaga (M. liza). Estos dos han presentado los datos de $\delta^{15} \mathrm{~N}$ y TL semejantes (prueba $\mathrm{U}, P>0,50$ ).

Los datos del isótopo ${ }^{15} \mathrm{~N}$ señalan, que entre los peces con hábito alimentario planctófago, la especie $S$. brasiliensis prefirió alimentarse del zooplancton, mientras la especie $M$. liza del fitoplancton (Tabla 1). 


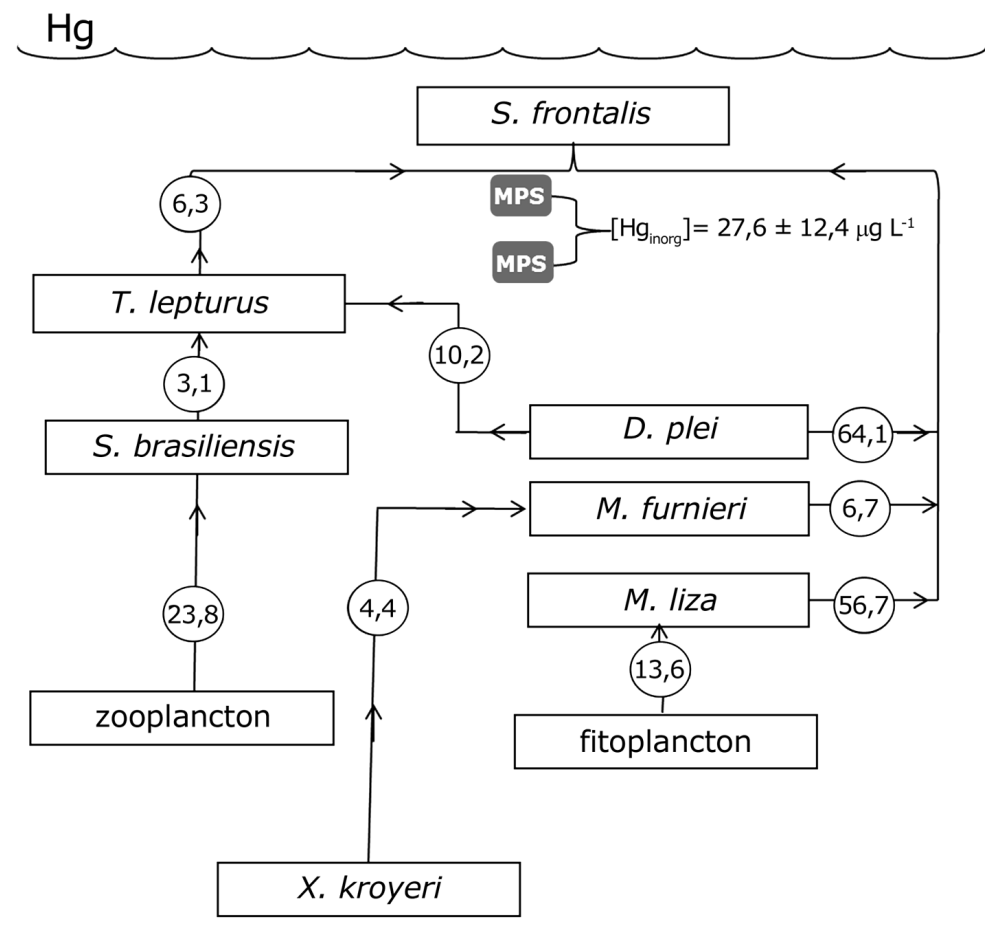

Sedimentos de fondo $\Rightarrow[\mathrm{MeHg}]=84,8 \pm 39,4 \mu \mathrm{kg}^{-1}$ peso seco

Figura 2. Los factores de biomagnificación (FBM) del Hga lo largo de la cadena trófica (los números señalados se refieren al FBM ), la concentración del $\mathrm{Hg}$ inorgánico $\left(\mathrm{Hg}_{\text {inorg }}\right)$ en el material particulado en suspensión en la columna de agua (MPS) y la concentración del metilmercurio (MeHg) en los sedimentos de fondo marino / Biomagnification factors (BMF) of $\mathrm{Hg}$ through a trophic chain (indicated numbers refer to BMF), inorganic $\mathrm{Hg}$ concentration $\left(\mathrm{Hg}_{\text {inorg }}\right)$ in suspended particulate matter in water column (SPM) and methylmercury (MeHg) concentration in seafloor sediments

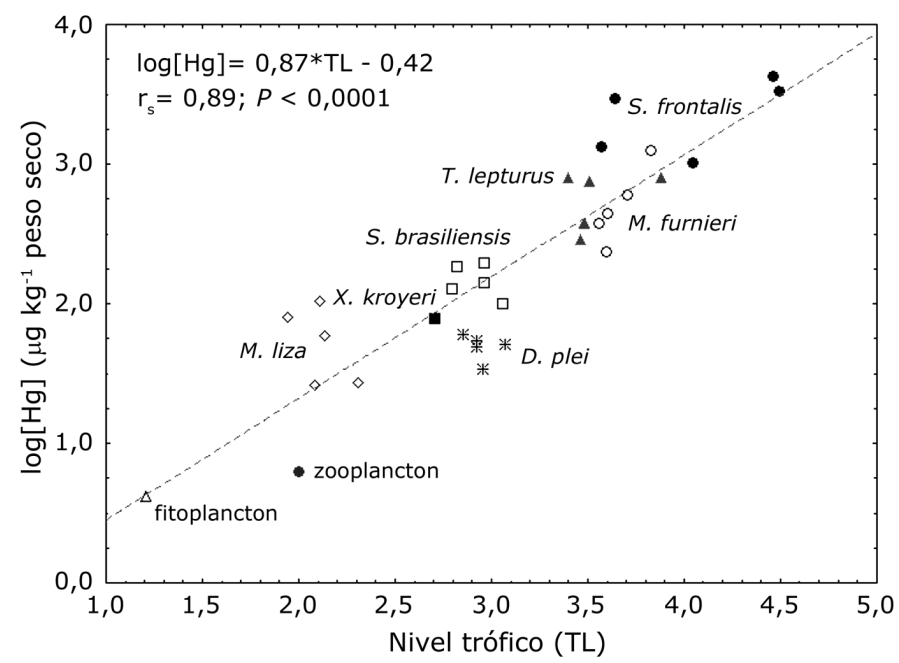

Figura 3. Relación entre la concentración de $\mathrm{Hg}\left(\log _{10}\right)$ y los valores del nivel trófico (TL) para todos los organismos que componen la cadena trófica / Relationship between $\mathrm{Hg}$ concentration $\left(\log _{10}\right)$ and trophic level (TL) for all organisms that compose the trophic chain 
Se encontró una correlación significativamente positiva $(\mathrm{r}=$ 0,89; $P<0,0001)$ entre las concentraciones musculares del $\mathrm{Hg}$ (en logaritmos) y el $\delta^{15} \mathrm{~N}$, incluyendo todos los ejemplares de las especies estudiadas. La pendiente $(\beta=0,26)$ de la regresión lineal simple $\left(\log [\mathrm{Hg}]=0,26 * \delta^{15} \mathrm{~N}-0,73\right)$ indica la magnificación trófica o el poder de biomagnificación del Hg en la cadena trófica investigada.

Se encontró una correlación significativamente positiva $\left(\mathrm{r}_{\mathrm{s}}=\right.$ $0,89 ; P<0,0001)$ entre el logaritmo de las concentraciones musculares del $\mathrm{Hg}$ y el TL, incluyendo todos los ejemplares de las especies estudiadas (Fig. 3). La pendiente $(\beta=0,87)$ de la regresión lineal simple en la misma figura fue usada para calcular el factor de magnificación trófica (FMT), cuyo valor fue de 7,44 , o sea, bastante mayor que uno, indicando que el $\mathrm{Hg}$ se biomagnifica en la cadena trófica.

\section{Discusión}

Aunque los estudios en relación con los contaminantes en los mamíferos marinos (centrándose en su uso como centinelas de la salud de los ecosistemas marinos y bioindicadores ambientales de la contaminación, y también en la toxicocinética de los metales) han aumentado desde las últimas décadas, cabe destacar que la especie $S$. frontalis ha sido muy poco estudiada. Los datos relativos sobre la acumulación de metales en el tejido hepático del Delfín Moteado del Atlántico son escasos y fragmentados (Kunito et al. 2004, Seixas et al. 2009, Lemos et al. 2013), y la presente investigación contribuye con uno de los primeros resultados de $\mathrm{Hg}$ en su tejido muscular (Fielding \& Evans 2014).

Normalmente, el Hg en el tejido muscular de diferentes especies de mamíferos marinos se encuentra casi en su totalidad en la forma de $\mathrm{MeHg}$ (Dietz et al. 1990, Chen et al. 2002). El estudio con los delfines del Caribe, incluyendo la especie del Delfín Moteado del Atlántico, ha verificado que aproximadamente el $80 \%$ del $\mathrm{Hg}$ total presente en el tejido muscular estaba en la forma de $\mathrm{MeHg}$ (Fielding \& Evans 2014). Además, en un estudio previo realizado con 2 pequeños cetáceos, Sotalia guianensis y Pontoporia blainvillei, de la costa norte de Río de Janeiro, el Hg muscular se encontraba en su mayoría como $\mathrm{MeHg}$ (el 94,4 y el 95,2\%, respectivamente) (Kehrig et al. 2016).

En general, los mamíferos marinos no presentan diferencias en la concentración de $\mathrm{Hg}$ relacionada con el sexo (O' Shea 1999, Baptista et al. 2016, Kehrig et al. 2016, Noël et al. 2016), como se ha verificado para el Delfín Moteado del Atlántico en este estudio.
Comúnmente, la longitud se relaciona positivamente con el Hg en los tejidos (muscular, hepático y renal) de los mamíferos marinos, hecho que fue verificado tanto en esta investigación para el músculo del Delfín Moteado del Atlántico, como en estudios previos con otras especies: Pontoporia blainvillei (Gerpe et al. 2002, Seixas et al. 2007), Cephalorhynchus c. commersonii (Cácerez-Saez et al. 2013), Sotalia guianensis (Kehrig et al. 2013), Tursiops truncatus (Monteiro et al. 2016) y Phoca vitulina (Noël et al. 2016). El Hg va acumulándose en los tejidos de los mamíferos a lo largo de su vida, o sea, la concentración aumenta con la longitud o la edad (Ikemoto et al. 2004, Noël et al. 2016). Esta alza en la concentración probablemente es el resultado de la acumulación continua del $\mathrm{Hg}$ a través de la dieta combinada con una eliminación lenta y también, una media vida biológica larga en estos animales (Neathery \& Miller 1975). Sin embargo, la vida media biológica del $\mathrm{Hg}$ en el organismo depende estrechamente de muchas variables que interactúan en los procesos de almacenamiento en nivel celular y también, sobre las transferencias entre órganos (Andre et al. 1991), una vez que este elemento posee una fuerte afinidad con el grupo $\mathrm{SH}$, presente en la cisteína (Kunito et al. 2004).

También, se ha atribuido el aumento en las concentraciones del $\mathrm{Hg}$ en los tejidos de los cetáceos, en las diferentes etapas de su vida (feto, cría, juvenil y adulto), a las diferentes formas de exposición, que cambian a lo largo del tiempo. El Hg puede ser transferido vía transferencia placentaria o lactacional de la madre a los fetos y las crías lactantes, afectándolos durante sus períodos más sensibles de desarrollo (Gerpe et al. 2002, Baptista et al. 2016, Noël et al. 2016). Cabe señalar que el $\mathrm{Hg}$, como MeHg, atraviesa la barrera placentaria y se acumula en los tejidos fetales de los mamíferos marinos, mientras que la placenta es una barrera importante contra el Hg inorgánico (Neathery \& Miller 1975). Además, las concentraciones del Hg en los tejidos de los cetáceos jóvenes y adultos, incluyéndose el Delfín Moteado del Atlántico, reflejan el contenido de $\mathrm{MeHg}$ de sus presas preferenciales, como los peces depredadores y los cefalópodos, ya que el $\mathrm{Hg}$ en la forma orgánica es la principal forma del $\mathrm{Hg}$ acumulado en los tejidos de las presas (Magalhães et al. 2007, Seixas et al. 2014).

Gracias al $\mathrm{Hg}$ en su forma orgánica, este elemento biomagnifica muy rápidamente a lo largo de las cadenas o redes tróficas, lo que lleva a los mayores depredadores, como los cetáceos, en los ecosistemas marinos, a acumularlo en elevados niveles en sus tejidos. Así siendo, las concentraciones de Hg en los peces depredadores voraces y en los cetáceos están fuertemente influenciadas por la estructura de la cadena y red trófica (Kidd et al. 2003) y por la longitud de la cadena trófica (Cabana \& Rasmussen 1994). 
Según los datos de $\mathrm{Hg}$ en la cadena trófica (Tabla 1), el delfín, $S$. frontalis es la especie más sensible al $\mathrm{Hg}$, una vez que asimila y acumula este elemento en el tejido muscular a través de la alimentación durante toda su larga vida, como fue reportado en estudios previos con otra especie de delfín, Sotalia guianensis, en la costa del Estado de Río de Janeiro (Bisi et al. 2012, Kehrig et al. 2013).

En esta investigación, los factores de biotransferencia (FBT) (Fig. 2) fueron calculados para comparar las posibles transferencias tróficas del $\mathrm{Hg}$ entre la presa (fuente) y sus consumidores, a lo largo de la cadena trófica costera, bajo la influencia de los eventos de surgencia. Vale la pena destacar que todos los valores de FBT fueron positivos, lo que indica que el $\mathrm{Hg}$ se transfirió con facilidad en todos los vínculos de la cadena trófica, desde su nivel más bajo, los plánctones, pasando por el camarón, el cefalópodo y los peces, hasta el depredador superior, el delfín. Además, estos hallazgos son corroborados con el FMT, cuyo valor calculado de 7,44, es bastante mayor que 1 , confirmando que el $\mathrm{Hg}$ biomagnifica a lo largo de toda la cadena trófica costera, desde los plánctones hasta el delfín.

En el estudio previo, en la costa norte del Estado de Río de Janeiro, que es una región de transición faunística, bajo la influencia del Río Paraíba del Sur, todos los FBT del Hg encontrados a lo largo de la cadena trófica costera fueron mayores que 1 (Kehrig et al. 2013), pero menores que los de la citada investigación. Así, el FBT entre la fuente alimenticia principal, el pez depredador voraz (T. lepturus) yel depredador superior (S. guianensis) presentó un valor de 2,4 (Kehrig et al. 2013), que es 2,6 menor que el FBT encontrado en el presente estudio entre el T. lepturus y el Delfín Moteado del Atlántico (Fig. 2).

El nivel trófico (TL) y la composición del isótopo estable $\delta^{15} \mathrm{~N}$ en el tejido muscular del Delfín Moteado del Atlántico y en el pez depredador voraz, T. lepturus, señala que estos dos depredadores están en niveles tróficos distintos (Tabla 1). Esta diferencia no era esperada, esto porque ambos predadores se alimentan con las mismas presas (Bittar \& Di Beneditto 2009, Melo et al. 2010, Lopes et al. 2012). Sin embargo, el delfín es una especie considerada teotófaga e ictiófaga (Lopes et al. 2012), mientras los adultos del pez son ictiófagos (Martins et al. 2005). En esta investigación se debe destacar el uso exclusivo de ejemplares adultos de T. lepturus.

En el estudio conducido en la costa norte del Estado de Río de Janeiro, se ha verificado que los depredadores S. guianensis y T. lepturus, estaban en niveles tróficos semejantes, ya que estas 2 especies también consumen las mismas presas en la alimentación y, consecuentemente, hay una analogía en la composición del isótopo estable del $\delta^{15} \mathrm{~N}$ en sus tejidos musculares (Kehrig et al. 2013), y consecuentemente, están en el mismo TL. Mientras tanto, en una investigación conducida en 3 bahías diferentes del Estado de Río de Janeiro, los datos de $\delta^{15} \mathrm{~N}$ mostraron que el delfín, S. guianensis, presentó valores más ligeros que los que se encontraron en el pez, T. lepturus recogidos en la Bahía de Sepetiba y en la Bahía de Ilha Grande. Por otro lado, en la Bahía de Guanabara, los valores de $\delta^{15} \mathrm{~N}$ en los ejemplares del cetáceo resultaron más pesados que los encontrados en el pez (Bisi et al. 2012).

En el estudio previo elaborado en la costa central del Estado de Río de Janeiro, el tejido muscular del Delfín Moteado del Atlántico presentó los mismos valores de $\delta^{15} \mathrm{~N}$ en el rango (12,2-15,4\%o) (Bisi et al. 2013) encontrado en la presente investigación. Según este estudio, este patrón obtenido de $\delta^{15} \mathrm{~N}$ para esta especie de delfín puede ser atribuido a la gran influencia de las masas de la corriente Agua Central del Atlántico Sur (Bisi et al. 2013).

Por lo tanto, distinguir los niveles tróficos con relación a los depredadores de una cadena trófica marina, que se alimentan con fuentes de recursos semejantes, es una tarea muy difícil, hecho que fue constatado tanto en esta investigación para el Delfín Moteado del Atlántico y el T. lepturus, como en los estudios previos citados anteriormente (Bisi et al. 2012, 2013; Kehrig et al. 2013)

La ausencia de una relación significativa entre los valores de $\delta^{15} \mathrm{~N}$ y la longitud en el Delfín Moteado del Atlántico, señaló que este cetáceo no ha cambiado su posición trófica a pesar de que la longitud pasó de 166 a 200 cm. Sin embargo, este resultado está coherente, ya que solamente se tomaron ejemplares inmaduros del delfín; hecho que fue comprobado en una investigación previa con otra especie de cetáceo (Kehrig et al. 2013). Las especies depredadoras voraces de una cadena o red trófica, que ocupan la misma posición trófica a lo largo de su vida, como el Delfín Moteado del Atlántico, están expuestas a concentraciones de $\mathrm{Hg}$ relativamente constantes en su alimentación. Lo que sugiere que la variabilidad del $\mathrm{Hg}$ entre los individuos puede estar vinculada a las diferencias en la posición trófica de cada ejemplar, independientemente de su tamaño (Bowles et al. 2001).

La composición del isótopo estable de nitrógeno en el tejido muscular del depredador refleja la concentración de ${ }^{15} \mathrm{~N}$ en la presa consumida a largo plazo a través de la nutrición. En esta investigación, efectivamente existe un enriquecimiento de $\delta^{15} \mathrm{~N}$ a lo largo de la cadena trófica, ya que los ejemplares de todas las especies juntas presentaron los valores desde 5,2\%o hasta $16,4 \%$ o (Tabla 1). Sin embargo, el valor de enriquecimiento de ${ }^{15} \mathrm{~N}$ a través de un nivel trófico a otro fue menor que lo previsto (3-4\%o) por De Niro \& Epstein (1981), hecho que 
también fue verificado en estudios previos con otras cadenas y redes tróficas de regiones costeras (Lavoie et al. 2010, Bisi et al. 2012, Di Beneditto et al. 2012, Kehrig et al. 2013). Las variaciones en la composición del $\delta^{15} \mathrm{~N}$ ayudan a elucidar los vínculos entre los niveles tróficos, así como las relaciones entre la captación del contaminante, en este estudio es el Hg, y la posición o nivel trófico de los ejemplares de las distintas especies (Cabana \& Rasmussen 1994).

En este estudio, la pendiente $(\beta=0,26)$ de la regresión lineal simple entre el logaritmo de las concentraciones del $\mathrm{Hg}$ y el isotopo estable del $\delta^{15} \mathrm{~N}$, que representa el grado total de la biomagnificación del $\mathrm{Hg}$ (Lavoie et al. 2013), ha mostrado que la cadena trófica costera tropical presentó un poder de biomagnificación para el $\mathrm{Hg}$ dentro de lo que se encuentra en el reportado en estudios previos en los diversos ecosistemas tropicales marinos $(0,16 \pm 0,08)$ y en ecosistemas costeros $(0,19 \pm 0,08)$ (Lavoie et al. 2013), así como, en la costa de Río de Janeiro (Brasil) (Bisi et al. 2012, Di Beneditto et al. 2012, Kehrig et al. 2013), el Golfo de San Lorenzo (Canadá) (Lavoie et al. 2010), el Norte del Golfo de México (México) (Senn et al. 2010), el Delta del Mekong (Vietnan) (Ikemoto et al. 2008), el Golfo de Omán (Sultanato de Omán) (Al-Reasi et al. 2007) y el lago Murray (Papúa Guinea) (Bowles et al. 2001).

La relación significativa encontrada entre el $\mathrm{Hg}$ y el $\delta^{15} \mathrm{~N}$ muscular sugiere que las preferencias de forrajeo individuales permanecen relativamente constantes durante períodos prolongados, lo que indica un grado de adherencia a la dieta y a las áreas de alimentación, como se ha encontrado en lo reportado en estudios previos (Di Beneditto et al. 2012, Kehrig et al. 2013).

Según Lavoie et al. (2013), el proceso de biomagnificación no se ve solamente afectado por el proceso de la cadena trófica (la tasa de crecimiento, la diversidad de especies y la longitud de la cadena trófica), sinotambién, por variables fisicoquímicas ambientales. Por lo tanto, la biomagnificación del $\mathrm{Hg}$ también ha sido evaluada con el FMT. Por lo tanto, este es una herramienta más confiable para evaluar y cuantificar la biomagnificación del $\mathrm{Hg}$ mediante su transferencia, a través de la dieta, para los consumidores a lo largo de la cadena trófica (Borgå et al. 2012, Lavoie et al. 2013).

El FMT calculado en la costa central del Estado de Río de Janeiro, es aproximadamente 1,4 veces mayor que lo encontrado en el estudio previo, en la costa norte del Estado de Río de Janeiro (FMT= 5,37) (Kehrig et al. 2013). Además, el FMT calculado $(4,87)$ en una red trófica Ártica (Jæger et al. 2009) ha presentado un valor menor que el de este estudio $(7,44)$. No obstante, estos datos han mostrado cómo la calidad ambiental y la biodisponibilidad del $\mathrm{Hg}$ pueden influir en su asimilación y en la acumulación por las especies marinas, y consecuentemente influir en el proceso de biomagnificación.

Según los datos encontrados en este estudio, las diferencias observadas en el proceso de biomagnificación del $\mathrm{Hg}$ en la cadena trófica tropical de la costa central del Estado de Río de Janeiro y en los diversos ecosistemas mundiales (costeros y tropicales) sugieren que las características particulares de cada ambiente y también, la estructura trófica de cada cadena alimenticia influyen en la intensidad de biomagnificación. Por lo tanto, siendo así, es muy difícil compararlos.

\section{Agradecimientos}

Los autores muestran gratitud al Consejo Nacional de Investigación de Brasil (CNPq), la Coordinación de Perfeccionamiento de Personal de Nivel Superior (CAPES, número de proyecto BEX 0128 / 14-7) y a la Fundación de Amparo a la Investigación del Estado de Río de Janeiro (FAPERJ) por el apoyo financiero. Agradecemos a Elisa Laporte Bomfim (Casa de España, Río de Janeiro) por su asistencia con relación a la edición del español.

Declaración ética: El método de recogida de datos, que incluye los animales varados muertos, está de acuerdo con el protocolo y las regulaciones establecidas por la Red de Varamientos Brasileña de Mamíferos Acuáticos (REMAB), coordinado por el Centro Nacional de Investigación y Conservación de Mamíferos Acuáticos (CMA-ICMBio) del Ministerio Brasileño del Medio Ambiente (MMA) <http://www.icmbio.gov.br/cma〉. Este estudio ha sido aprobado por el Instituto Chico Mendes para la Conservación de la Biodiversidad (ICMBio), y fue conducido según la licencia \# 32550-2 del SISBIO.

\section{LITERATURA CITADA}

Alava JJ \& FAPC Gobas. 2012. Assessing biomagnification and trophic transport of persistent organic pollutants in the food chain of the Galapagos sea lion (Zalophus wollebaeki): conservation and management implications. In: Romero A \& EO Keith (eds). New approaches to the study of marine mammals, pp. 77-108. InTech Publisher, Rijeka.

Al-Reasi HA, FA Ababneh \& DR Lean. 2007. Evaluating mercury biomagnification in fish from a tropical marine environment using stable isotopes $\left(\delta^{13} \mathrm{C}\right.$ and $\left.\delta^{15} \mathrm{~N}\right)$. Environmental Toxicology and Chemistry 26: 1572-1581.

Amaral KB, DJ Alvares, L Heinzelmann, M BorgesMartins, S Siciliano \& IB Moreno. 2015. Ecological niche modeling of Stenella dolphins (Cetartiodactyla: Delphinidae) in the southwestern Atlantic Ocean. Journal of Experimental Marine Biology and Ecology 472: 166-179.

Andre J, A Boudou, F Ribeyre \& M Bernhard. 1991. Comparative study of mercury accumulation in dolphins (Stenella coeruleoalba) from French Atlantic and Mediterranean coasts. Science of the Total Environment 104: 191-209. 
Aubail A, J Teilmann, R Dietz, F Rigét, T Harkonen, O Karlsson, A Rosing-Asvid \& F Caurant. 2011. Investigation of mercury concentrations in fur of phocid seals using stable isotopes as tracers of trophic levels and geographical regions. Polar Biology 34(9): 1411-1420.

Baeyens W, M Leermakers, T Papina, A Saprykin, N Brion, J Noyen, M De Gieter, M Elskens \& L Goeyens. 2003. Bioconcentration and biomagnification of mercury and methylmercury in North Sea and Scheldt Estuary fish. Archives of Environmental Contamination and Toxicology 45: 498-508.

Baptista G, HA Kehrig, APM Di Beneditto, RA HauserDavis, MG Almeida, CE Rezende, S Siciliano, JF de Moura \& I Moreira. 2016. Mercury, selenium and stable isotopes in four small cetaceans from the Southeastern Brazilian coast: influence of feeding strategy. Environmental Pollution 218: 1298-1307.

Barwick M \& W Maher. 2003. Biotransference and biomagnification of selenium, copper, cadmium, zinc, arsenic and lead in a temperate seagrass ecosystem from Lake Macquarie Estuary, NSW, Australia. Marine Environmental Research 56: 471-502.

Bisi TL, G Lepoint, AF Azevedo, PR Dorneles, L Flach, K Das, O Malm \& J Lailson-Brito. 2012. Trophic relationships and mercury biomagnification in Brazilian tropical coastal food webs. Ecological Indicators 18: 291-302.

Bisi TL, PR Dorneles, J Lailson-Brito, G Lepoint, AF Azevedo, L Flach, O Malm \& K Das. 2013. Trophic relationships and habitat preferences of delphinids from the southeastern Brazilian coast determined by carbon and nitrogen stable isotope composition. PLoS ONE 8(12): e82205. <https:/ /doi.org/10.1371/journal.pone.0082205>

Bittar VT \& APM Di Beneditto. 2009. Diet and potential overlap between Trichiurus lepturus (Osteichthyes: Perciformes) and Pontoporia blainvillei (Mammalia: Cetacea) in northern Rio de Janeiro, Brazil. Zoologia 26(2): 374-378.

Borgå K, KA Kidd, DCG Muir, O Berglund, JM Conder, FAPC Gobas, J Kucklick, O Malm \& DE Powell. 2011. Trophic magnification factors: considerations of ecology, ecosystems, and study design. Integrated Environmental Assessment and Management 8(1): 64-84.

Bowles KC, SC Apte, WA Maher, M Kawei \& R Smith. 2001. Bioaccumulation and biomagnification of mercury in Lake Murray, Papua New Guinea. Canadian Journal of Fisheries and Aquatic Sciences 58: 888-897.

Broman D, C Naf, C Rolff, Y Zebuhr, B Fry \& J Hobbie. 1992. Using ratios of stable nitrogen isotopes to estimate bioaccumulation and flux of polychlorinated dibenzo-pdioxins (PCDDs) and dibenzofurans (PCDFs) in two food-chains from the northern Baltic. Environmental Toxicology and Chemistry 11:331-345.
Cabana G \& JB Rasmussen. 1994. Modelling food chain structure and contaminant bioaccumulation using stable nitrogen isotopes. Nature 372: 255-373.

Cáceres-Saez I, NA Dellabianca, RNP Goodall, HL Cappozzo \& SR Guevara. 2013. Mercury and selenium in subantarctic Commerson's Dolphins (Cephalorhynchus c.commersonii). Biological Trace Element Research 151(2): 195-208.

Chen M, C Shih, C Chou \& I Chou. 2002. Mercury, organicmercury and selenium in small cetaceans in Taiwanese waters. Marine Pollution Bulletin 89:30-34.

Coelho-Souza SA, MS López, JRD Guimarães, R Coutinho \& RN Candella. 2012. Biophysical interactions in the Cabo Frio upwelling system, Southeastern Brazil. Brazilian Journal of Oceanography 60(3): 353-365.

Das K, L Holsbeek, J Browning, U Siebert, A Birkun Jr \& JM Bouquegneau. 2004. Trace metal and stable isotope measurements $\left(\delta^{13} \mathrm{C}\right.$ and $\left.\delta^{15} \mathrm{~N}\right)$ in the harbor porpoise Phocoena phocoena relicta from the Black sea. Environmental Pollution 131: 197-204.

De Niro MJ \& S Epstein. 1981. Influence of diet on the distribution of nitrogen isotopes in animals. Geochimica et Cosmochimica Acta 45: 341-351.

Di Beneditto APM, RMA Ramos, S Siciliano, RA Santos, G Bastos \& E Fagundes-Netto. 2001. Stomach contents of dolphins from Rio de Janeiro, South-eastern Brazil. Aquatic Mammals 27(1): 24-28.

Di Beneditto APM, CMM Souza, HA Kehrig \& CE Rezende. 2011. Use of multiple tools to assess the feeding preference of coastal dolphins. Marine Biology 158: 22092217.

Di Beneditto APM, VT Bittar, PB Cardoso, CE Rezende \& HA Kehrig. 2012. Mercury and nitrogen isotope in a marine species from a tropical coastal food web. Archives of Environmental Contamination Toxicology 62: 264-271.

Dietz R, CO Nielsen, MM Hansen \& CT Hanse. 1990. Organic mercury in Greenland birds and marine mammals. Science of the Total Environment 95: 41-51.

FAO/SIDA. 1983. Manual de métodos de investigación del medio ambiente acuático. Parte 9. Análisis de presencia de metales y organoclorados en los peces. FAO Documento Técnico de Pesca 212: 1-35.

Fielding R \& DW Evans. 2014. Mercury in Caribbean dolphins (Stenella longirostris and Stenella frontalis) caught for human consumption off St. Vincent, West Indies. Marine Pollution Bulletin 89: 30-34.

Gerpe MS, DH Rodríguez, VJ Moreno, RO Bastida \& JAE de Moreno. 2002. Accumulation of heavy metals in the franciscana (Pontoporia blainvillei) from Buenos Aires Province, Argentina. The Latin American Journal of Aquatic Mammals 1(1): 95-106. 
Ikemoto T, T Kunito, I Watanabe, G Yasunaga, N Baba, N Miyazaki, EA Petrov \& S Tanabe. 2004. Comparison of trace element accumulation in Baikal seals (Pusa sibirica), Caspian seals (Pusa caspica) and northern fur seals (Callorhinus ursinus). Environmental Pollution 127: 83-97.

Ikemoto T, NPC Tu, N Okuda, A Iwata, K Omori, S Tanabe, BC Tuyen \& I Takeuchi. 2008. Biomagnification of traceelements in the aquatic food web in the Mekong delta, South Vietnam using stable carbon and nitrogen isotope analysis. Archives of Environmental Contamination and Toxicology 54: 504-515.

Jæger I, H Hop \& GW Gabrielsen. 2009. Biomagnification of mercury in selected species from an Arctic marine food web in Svalbard. Science of the Total Environment 407: 47444751.

Jardine TD, KA Kidd \& AT Fisk. 2006. Applications, considerations, and sources of uncertainty when using stable isotope analysis in ecotoxicology. Environmental Science and Technology 40(24): 7501-7511.

Kehrig HA, TG Seixas, EA Palermo, AP Baêta, CW Castelo-Branco, O Malm \& I Moreira. 2009a. The relationships between mercury and selenium in plankton and fish from a tropical food web. Environmental Science Pollution Research 16: 10-24.

Kehrig HA, KWG Fernandes, O Malm, TG Seixas, APM Di Beneditto \& CM Souza. 2009b. Transferência trófica de mercúrio e selênio na costa norte do Rio de Janeiro. Química Nova 32: 1822-1828.

Kehrig HA, TG Seixas, O Malm, APM Di Beneditto \& CE Rezende. 2013. Mercury and selenium biomagnification in a Brazilian coastal food web using nitrogen stable isotope analysis: a case study in an area under the influence of the Paraiba do Sul River plume. Marine Pollution Bulletin 75: 283-290.

Kehrig HA, RA Hauser-Davis, TG Seixas, AB Pinheiro \& APM Di Beneditto. 2016. Mercury species, selenium, metallothioneins and glutathione in two dolphins from the southeastern Brazilian coast: mercury detoxification and physiological differences in diving capacity. Environmental Pollution 213: 785-792.

Kidd K, HA Bootsma, RH Heslein, WL Lockhart \& RE Hecky. 2003. Mercury concentrations in food web of Lake Malawi, East Africa. Journal of Great Lakes Research 29: 258-266.

Kunito T, S Nakamura, T Ikemoto, Y Anan, R Kubota, S Tanabe, FCW Rosas, G Fillmann \& JW Readman. 2004. Concentration and subcellular distribution of trace elements in liver of small cetaceans incidentally caught along the Brazilian coast. Marine Pollution Bulletin 49: 574-587.

Lavoie RA, CE Hebert, J-F Rail, BM Braune, E Yumvihoze, LG Hill \& DRS Lean. 2010. Trophic structure and mercury distribution in a Gulf of St. Lawrence (Canada) food web using stable isotope analysis. Science of the Total Environment 408:5529-5539.
Lavoie RA, TD Jardine, MM Chumchal, KA Kidd \& LM Campbell. 2013. Biomagnification of mercury in aquatic food webs: a worldwide meta-analysis. Environmental Science \& Technology 47: 13385-13394.

Lemos LS, JF de Moura, RA Hauser-Davis, RC Campos \& S Siciliano. 2013. Small cetaceans found stranded or accidentally captured in southeastern Brazil: Bioindicators of essential and non-essential trace elements in the environment. Ecotoxicology and Environmental Safety 97: 166-175.

Lopes XM, MCO Santos, E Silva, M Bassoi \& RA Santos. 2012. Feeding habits of the Atlantic Spotted Dolphin, Stenella frontalis, in southeastern Brazil. Brazilian Journal of Oceanography 60(2): 189-198.

Magalhães MC, V Costa, GM Menezes, MR Pinho, RS Santos \& LR Monteiro. 2007. Intra- and inter-specific variability in total and methylmercury bioaccumulation by eight marine fish species from the Azores. Marine Pollution Bulletin 54: $1654-1662$

Martins AS, M Haimovici \& R Palacios. 2005. Diet and feeding of the cutlassfish Trichiurus lepturus in the Subtropical Convergence Ecosystem of southern Brazil. Journal of the Marine Biological Association of the United Kingdom 85: 1223-1229.

Melo CLC, RA Santos, M Bassoi, AC Araujo, J LailsonBrito, PR Dorneles \& AF Azevedo. 2010. Feeding habits of dolphins (Mammalia: Cetacea) from Rio de Janeiro State, Brazil. Journal of the Marine Biological Association of the United Kingdom 90(8): 1509-1515.

Monteiro SS, J Torres, M Ferreira, A Marçalo, L Nicolau, JV Vingada \& C Eira. 2016. Ecological variables influencing trace element concentrations in bottlenose dolphins (Tursiops truncatus, Montagu 1821) stranded in continental Portugal. Science of the Total Environment 544: 837-844.

Mullin KD \& GL Fulling. 1998. Abundance of cetaceans in the southern U.S. North Atlantic Ocean during summer. Fishery Bulletin 101: 603-613.

Neathery MW \& WL Miller. 1975. Metabolism and toxicity of cadmium, mercury, and lead in animals: a review. Journal of Dairy Science 58(12): 1767-1781.

Noël M, S Jeffries, DM Lambourn, K Telmer, R Macdonald \& PS Ross. 2016. Mercury accumulation in Harbour seals from the Northeastern Pacific Ocean: the role of transplacental transfer, lactation, age and location. Archives of Environmental Contamination and Toxicology 70: 56-66.

O'Shea TJ. 1999. Environmental contaminants and marine mammals. In: Reynolds JE III \& SA Rommel (eds). Biology of marine mammals, pp. 485-563. Amiksonian Institution Press, Washington.

Panebianco MV, MF Negri, SE Botté, JE Marcovecchio \& HL Cappozzo. 2011. Metales pesados en el riñón del delfín franciscana, Pontoporia blainvillei (Cetacea: Pontoporiidae) y su relación con parámetros biológicos. Latin American Journal of Aquatic Research 39(3): 526-533. 
Peterson BJ \& B Fry. 1987. Stable isotopes in ecosystem studies. Annual Review of Ecology, Evolution, and Systematics 18: 293-320.

Seixas TG, HA Kehrig, G Fillmann, APM Di Beneditto, CMM Souza, ER Secchi, I Moreira \& O Malm. 2007. Ecological and biological determinants of trace elements accumulation in liver and kidney of Pontoporia blainvillei. Science of the Total Environment 385: 208-220.

Seixas TG, HA Kehrig, APM Di Beneditto, CMM Souza, O Malm \& I Moreira. 2009. Trace elements in different species of cetacean from Rio de Janeiro coast. Journal of the Brazilian Chemical Society 20: 243-251.

Seixas TG, I Moreira, S Siciliano, O Malm \& HA Kehrig. 2014. Differences in methylmercury and inorganic mercury biomagnification in a tropical marine food web. Bulletin of Environmental Contamination and Toxicology 92(3): 274-278.

Senn DB, EJ Chesney, JD Blum, MS Bank, A Maage \& JP Shine. 2010. Stable isotope $(\mathrm{N}, \mathrm{C}, \mathrm{Hg})$ study of methylmercury sources and trophic transfer in the Northern Gulf of Mexico. Environmental Science and Technology 44: 1630-1637.
Siciliano S, RM Ramos, APM Di Beneditto, MCO Santos, AB Fragoso, J Lailson-Brito Jr, AF Azevedo, AFC Vicente, E Zampirolli, FS Alvarenga, L Barbosa \& NR Lima. 2007. Age and growth of some delphinids in Southeastern Brazil. Journal of the Marine Biological Association of the United Kingdom 87:293-303.

UNEP. 2013. Minamata convention on mercury. Textos y Anexos. Interim Secretariat of the Minamata Convention on Mercury, United Nations Environment Programme, Geneva. <http://www.mercuryconvention.org/Portals/11/documents/ Booklets/Minamata Conventionon Mercury booklet Spanish.pdf>

Vander Zanden MJ, G Cabana \& JB Rasmussen. 1997. Comparing the trophic position of littoral fish estimated using stable nitrogen isotopes $\left(\delta^{15} \mathrm{~N}\right)$ and dietary data. Canadian Journal of Fishery and Aquatic Sciences 54(5): 1142-1158.

Recibido el 27 de julio del 2016 y aceptado el 7 de marzo de 2017

Editor: Claudia Bustos D. 